\title{
Comment on "Factors related to adherence to antiretroviral treatment in a specialized care facility"
}

\author{
Yuexiao $\mathrm{Yu}^{1}$, Xiaofei Li ${ }^{1 *}$
}

Dear Editor,

We are glad to read this very valuable article entitled "Factors related to adherence to antiretroviral treatment in a specialized care facility." They ${ }^{1}$ found that symptomatic patients have better adherence to therapy. The authors indicate that having aids decreases the probability of non-adherence to antiretroviral treatment by $92 \%$. But in my opinion, there are still some issues that should be raised.

First of all, this is a small sample study. So how does the author sample? Is the sample representative? The author should give a detailed introduction to the environment of the subject. What is the size of the population of the city, is the sample a whole group sample or a multi-stage sample? The representativeness of the sample is the basis for reaching reliable conclusions.

As the author says, in Brazil, a study conducted in 55 health services specialized in the care for patients with HIV/aids showed large variations in the non-adherence rates throughout the country, ranging from $10.7-86.0 \%$. Therefore, future research needs to pay attention to the representativeness of samples and large sample studies.

\section{REFERENCE}

1. Velame KT, Silva RSD, Cerutti Junior C. Factors related to adherence to antiretroviral treatment in a specialized care facility. Rev Assoc Med Bras (1992). 2020;66(3):290-5. https://doi.org/10.1590/1806-9282.66.3.290

'Yiwu Central Hospital, Department of infectious diseases - Zhejiang, China

*Corresponding author: xiaofeili2000@163.com

Conflicts of interest: the authors declare there is no conflicts of interest. Funding: none

Received on February 09, 2021. Accepted on March 14, 2021. 